\title{
Cyclic nigerosylnigerose ameliorates DSS-induced colitis with restoration of goblet cell number and increase in IgA reactivity against gut microbiota in mice
}

\author{
Takeshi TSURUTA ${ }^{1 *}$, Emiko KATSUMATA ${ }^{1 \dagger}$, Akiko MIZOTE ${ }^{2}$, Hou Jian JIAN ${ }^{1}$, Teresia Aluoch MUHOMAH ${ }^{1}$ \\ and Naoki NISHINO ${ }^{1}$ \\ ${ }^{1}$ Laboratory of Animal Nutrition, Graduate School of Environmental and Life Science, Okayama University, 1-1-1 Tsushimanaka, Kita-ku, \\ Okayama 700-8530, Japan \\ ${ }^{2}$ Hayashibara Co., Ltd., Okayama 702-8006, Japan
}

Received February 18, 2020; Accepted April 11, 2020; Published online in J-STAGE April 25, 2020

\begin{abstract}
Cyclic nigerosylnigerose (CNN) is a cyclic oligosaccharide. Oral administration of CNN promotes immunoglobulin A (IgA) secretion in the gut. IgA is a major antibody secreted into the gut and plays a crucial role in suppressing gut inflammation due to commensal gut microbiota. To investigate the effect of administration of CNN to promote IgA secretion on gut inflammation, experimental colitis was induced with dextran sulfate sodium (DSS) in Balb/c mice after 6 weeks of CNN pre-feeding. The severity of colitis was evaluated based on a disease activity index (DAI), the gene expression of inflammatory cytokines, and a histological examination. The CNN-treated mice with DSS-induced colitis (CNN-DSS group) showed significantly lower DAI scores and mRNA levels of interleukin-1 compared with the CNN-untreated mice with DSS-induced colitis (DSS group). Histological examination of the colon revealed that the pathological score was significantly lower in the CNN-DSS group compared with the DSS group due to the reduced infiltration of immune cells. The number of goblet cells was significantly higher in the CNN-DSS group compared with the DSS group. The IgA concentration and the ratio of microbiota coated with IgA were evaluated in the cecal content. Although there was no difference in the IgA concentration among groups, a higher proportion of cecal microbiota were coated with IgA in the CNN-DSS group compared with that in the DSS group. These results suggest that CNN might preserve goblet cells in the colon and promote IgA coating of gut microbiota, which synergistically ameliorate gut inflammation in mice with DSS-induced colitis.
\end{abstract}

Key words: oligosaccharide, DSS-induced colitis, goblet cell, gut microbiota, immunoglobulin A

\section{INTRODUCTION}

Inflammatory bowel diseases (IBDs) comprise a group of heterogeneous chronic and relapsing inflammatory gut disorders that include ulcerative colitis and Crohn's disease. Previous studies have suggested that IBDs are associated with an abnormal composition of gut microbiota. Compared with healthy individuals, several opportunistic pathogens and a smaller number of beneficial microbiota are found in the gut of IBD patients [1-3]. Moreover, depletion of gut microbiota by antibiotic treatment ameliorates clinical symptoms in IBD patients [2], suggesting that excessive inflammatory responses against gut microbiota are a major factor affecting the onset of gut inflammation. The use of dietary ingredients that can modulate the composition of gut microbiota and, therefore, suppress gut inflammation has recently become a focus of interest for the prevention of IBDs [4].
Cyclic nigerosylnigerose ( $\mathrm{CNN})$ is a cyclic oligosaccharide composed of four D-glucopyranosyl residues linked by alternating $\alpha-1,3$ and $\beta-1,6$ glucosidic linkages. CNN is found naturally in Japanese sake $(2.2 \mu \mathrm{g} / \mathrm{mL})$ and sake lees $(206 \mu \mathrm{g} / \mathrm{g})$ [5] and can also be synthesized from maltodextrins by a combined reaction of two transferases $[6,7]$. A previous study showed that oral administration of $\mathrm{CNN}$ increased the immunoglobulin $\mathrm{A}$ (IgA) concentration in mouse feces [8]. IgA, a major antibody isotype expressed in the gut, helps to maintain gut homeostasis [9-11]. One of the major functions of IgA is immune exclusion of pathogenic microbes by restricting access to the epithelial surface $[10,12]$. Furthermore, recent studies have shown that IgA plays a crucial role in suppressing gut inflammation due to commensal microbiota. Kubinak et al. showed that decreased IgA coating of gut microbiota in mice that are deficient in T cell MyD88 expression resulted in more severe gut inflammation following

${ }^{\dagger}$ Co-first author.*Corresponding author. Takeshi Tsuruta (E-mail: tsurutafe@okayama-u.ac.jp) (C2020 BMFH Press

This is an open-access article distributed under the terms of the Creative Commons Attribution Non-Commercial No Derivatives (by-nc-nd) License. (CC-BY-NC-ND 4.0: https://creativecommons.org/licenses/by-nc-nd/4.0/) 
2,4,6-trinitrobenzenesulfonic acid (TNBS)-induced colitis compared with that in wild-type mice [13]. Furthermore, Peterson et al. showed that IgA, which is specific for gut bacterial antigens, suppressed proinflammatory gut signaling [14]. Although we observed that oral administration of CNN enhanced IgA secretion in the gut, the role of the IgA secreted after $\mathrm{CNN}$ ingestion remains unclear. Since the IgA coating of gut microbiota helps regulate gut inflammation, we hypothesized that the stimulatory effect of CNN on IgA secretion in the gut might be useful for suppressing gut inflammation. To validate our hypothesis, we evaluated the effect of oral administration of CNN on symptoms of dextran sulfate sodium (DSS)-induced colitis in mice. Moreover, the ratio of IgAcoated microbiota in the cecal content was evaluated to understand whether $\mathrm{CNN}$-treated IgA coats gut microbiota.

\section{MATERIALS AND METHODS}

This study was approved by the Animal Care and Use Committee of Okayama University, Japan (approval no. OKU-2016399), and complies with the institutional and national guidelines for the care and use of laboratory animals.

\section{Animals and treatment}

Ten-week-old female Balb/c mice were obtained from Charles River Laboratories Japan (Yokohama, Japan). Mice were given a commercial pelleted diet (Labo MF stock, Nosan Corporation, Kanagawa, Japan) and were allowed free access to water. After a 7-day adaptation period, mice were divided into three groups: control group, DSS-induced colitis group (DSS group), and DSSinduced colitis with CNN treatment group (CNN-DSS group) $(n=5$ for each group). All groups were fed Labo MF stock ad libitum. CNN (Hayashibara Co., Ltd., Okayama, Japan) was added to water at a concentration of $22.5 \mathrm{mg} / \mathrm{mL}$ and given to CNN-DSS mice as their drinking water. The concentration of $\mathrm{CNN}$ used to promote gut IgA secretion was determined with reference to a previous study [8]. The mice were kept at room temperature $\left(25^{\circ} \mathrm{C}\right)$ under a controlled $12 \mathrm{hr}$ light/dark cycle. Induction of IgA secretion with $\mathrm{CNN}$ was performed as previously described [8]. Briefly, a six-week feeding period was set, after which feces were collected for measurement of the IgA concentration. After confirming that CNN significantly increased fecal IgA concentrations, colitis was induced by treatment with $1.5 \%(\mathrm{w} / \mathrm{v})$ DSS (MW $=1,000-9,000$, dissolved in drinking water; Wako Pure Chemical, Tokyo, Japan) in the DSS and CNN-DSS groups. The severity of DSS-induced colitis was assessed twice a week using a disease activity index (DAI) based on the scoring system shown in Table 1 [15], which scores based on loss of body weight, stool consistency, and rectal bleeding. DAI scores were assessed until 14 days post-DSS treatment. On day 14 post-DSS treatment, mice were anesthetized by intraperitoneal administration of pentobarbital $(30 \mathrm{mg} / \mathrm{kg})$ and euthanized by cervical dislocation. The colon was collected and its length was measured. The distal colon was cut into three segments for gene expression analysis, measurement of myeloperoxidase (MPO) activity, and histological examination. The cecal content was collected to evaluate microbial composition, $\operatorname{IgA}$ concentration, and $\operatorname{IgA}$ reactivity against gut microbiota. Independent animal experimentation was performed in duplicate.

\section{Gene expression of inflammatory cytokines in the colon}

The mRNA levels of inflammatory cytokines, interleukin- $1 \beta$
Table 1. Criteria for scoring disease activity index (DAI)*

\begin{tabular}{cccc}
\hline Score & Weight loss (\%) & Stool consistency** & Rectal bleeding \\
\hline 0 & None & Normal & Negative \\
1 & $1-5$ & Loose stool & Hemoccult positive \\
2 & $5-10$ & Diarrhea & Gross bleeding \\
3 & $10-15$ & & \\
4 & $>15$ & &
\end{tabular}

*DAI was calculated by summing the scores of weight loss, stool consistency, and rectal bleeding.

**Normal stools: well formed pellets; loose stool: pasty stool that does not stick to the anus; diarrhea: liquid stools that sticks to the anus.

(illb), interleukin-6 (ilo), and monocyte chemoattractant protein-1 ( $m c p 1$ ), were evaluated by quantitative real-time PCR. Total RNA was extracted from the distal colon using ISOGEN II, according to the manufacturer's protocol (Nippon Gene, Tokyo, Japan). Complementary DNA (cDNA) was synthesized from $500 \mathrm{ng}$ total RNA using ReverTra Ace with random primers (Toyobo, Osaka, Japan). The synthesized cDNA was mixed with primer pairs and GeneAce SYBR qPCR Mix $\alpha$ (Nippon Gene, Tokyo, Japan). Amplifications were performed in an AriaMx RealTime PCR System (Agilent Technologies Japan, Tokyo, Japan) using the following temperature profiles: one cycle at $95^{\circ} \mathrm{C}$ for $10 \mathrm{~min}$, followed by 40 cycles of denaturation at $95^{\circ} \mathrm{C}$ for $30 \mathrm{sec}$ and annealing at $60^{\circ} \mathrm{C}$ for $60 \mathrm{sec}$. Sample quantification cycle (Cq) values were normalized against that of the housekeeping gene, glyceraldehyde 3-phosphate dehydrogenase (gapdh). Fold changes compared with the control group were then calculated using the $2^{-\Delta \Delta \mathrm{Cq}}$ method. The primer pairs used were as follows: 5'-TCAAGAAGGTGGTGAAGCAG-3' and 5'-AGTGGGAGTTGCTGTTGAAGT-3' for gapdh, 5'-CAACCAACAAGTGATATTCTCCATG-3' and 5'-GATCCACACTCTCCAGCTGCA-3' for $i l 1 b, \quad 5 '-\mathrm{GAGGATACCACTCCCAACAGACC-3'}$ and 5'-AAGTGCATCATCGTTGTTCATACA-3' for il6, and 5'-CTTCTGGGCCTGCTGTTCA-3' and 5'CCAGCCTACTCATTGGGATCA-3' for $m c p 1$.

\section{MPO activity}

The MPO activity in the colon was assessed using a protocol described by Kim et al. [16]. In brief, frozen colonic tissue was homogenized in $50 \mathrm{mM}$ potassium phosphate buffer containing $13.7 \mathrm{mM}$ hexadecyltrimethylammonium bromide ( $\mathrm{pH}$ 6.0) with zirconia beads ( $2.3 \mathrm{~mm}$ diameter) using Beads Crusher $\mu \mathrm{T}-12$ $(1,800 \mathrm{rpm}, 2 \times 30 \mathrm{sec}$, Taitec, Tokyo, Japan $)$ and centrifuged at $4^{\circ} \mathrm{C}$ at $12,000 \times \mathrm{g}$ for $15 \mathrm{~min}$. Seven microliters of the supernatant was mixed with $200 \mu \mathrm{L}$ of $0.167 \%$ (w/v) o-dianisidine dihydrochloride (Sigma-Aldrich Japan, Tokyo, Japan) and $0.0006 \%(\mathrm{v} / \mathrm{v})$ hydrogen peroxide (Sigma-Aldrich Japan, Tokyo, Japan) solution. The protein concentration was measured using a commercial bicinchoninic acid kit according to the manufacturer's instructions (Nacalai Tesque, Kyoto, Japan). MPO activity was expressed as the change in absorbance at $450 \mathrm{~nm} / \mathrm{min} / \mathrm{mg}$ protein.

\section{Histological examination of colonic tissue}

The distal colon was cut and fixed with $10 \%$ neutral phosphatebuffered formalin (Wako Pure Chemical). The fixed colonic tissue was dehydrated, embedded in paraffin, and sectioned into 
5 - $\mu$ m-thick slices. For histological analysis, slices were stained with standard hematoxylin-eosin (H\&E) to evaluate mucosal inflammation. For mucus analysis, slices were stained with Alcian Blue ( $\mathrm{pH}$ 2.5) to evaluate them for the presence of goblet cells. The cross sections of colonic tissue specimens were examined at $200 \times$ magnification and imaged using an All-in-One Fluorescence Microscope (BZ-X710; Keyence, Osaka, Japan). Histological score was evaluated for eight microscopic fields of each sample using a semiquantitative scoring method, as shown in Table 2 [17]. The goblet cell number per crypt was counted for eight microscopic fields of each sample.

\section{Pyrosequencing of bacterial $16 S$ rRNA gene (V4) amplicon sequencing in the cecal content}

DNA was extracted from cecal content using a QIAamp stool mini kit (Qiagen Japan, Tokyo, Japan), according to the manufacturer's instructions, with additional freeze-thaw and bead-beating steps. The extracted DNA was subjected to two-step PCR reactions to generate amplicon libraries for next generation sequencing (NGS). In the first PCR reaction, primers targeting the V4 region of 16S rRNA genes (forward, 5'-ACACTCTTTCCCTACACG ACGCTCTTCCGATCTGTGCCAGCMGCCGCGGTAA-3'; reverse, 5'-GTGACTGGAGTTCAGACGTGTGCTCTTCCG ATCTGGACTACHVGGGTWTCTAAT-3'; tail sequences are underlined) were used. Amplification was performed using the following temperature profiles: one cycle at $94^{\circ} \mathrm{C}$ for $2 \mathrm{~min}$, followed by 25 cycles of denaturation at $94^{\circ} \mathrm{C}$ for $30 \mathrm{sec}$, annealing at $50^{\circ} \mathrm{C}$ for $30 \mathrm{sec}$, and extension at $72^{\circ} \mathrm{C}$ for $30 \mathrm{sec}$, and then one cycle at $72^{\circ} \mathrm{C}$ for $5 \mathrm{~min}$ for final extension. The PCR products were purified by electrophoretic separation on a $2 \%(\mathrm{w} / \mathrm{v})$ agarose gel using a Fast Gene Gel/PCR Extraction Kit (NIPPON Genetics Co., Ltd., Tokyo, Japan) and subjected to a second round of PCR with adapter-attached primers. Amplification was performed using the following temperature profiles: one cycle at $94^{\circ} \mathrm{C}$ for $2 \mathrm{~min}$, followed by 10 cycles of denaturation at $94^{\circ} \mathrm{C}$ for $30 \mathrm{sec}$, annealing at $59^{\circ} \mathrm{C}$ for $30 \mathrm{sec}$, and extension at $72^{\circ} \mathrm{C}$ for $30 \mathrm{sec}$, and then one cycle at $72^{\circ} \mathrm{C}$ for $5 \mathrm{~min}$ for final extension. PCR products were purified as described above for the first-round products. The purified amplicons were pair-end sequenced $(2 \times 250)$ on an Illumina MiSeq platform at FASMAC Co., Ltd. (Kanagawa, Japan).

\section{Bioinformatics}

The raw paired-end FASTQ reads were demultiplexed using idemp (https://github.com/yhwu/idemp/blob/master/idemp. cp) and imported into the Quantitative Insights Into Microbial Ecology 2 program (QIIME2, ver. 2017.9.0, https://qiime2.org/) [18]. The Divisive Amplicon Denoising Algorithm 2 (DADA2) [19] was used to quality filter, trim, de-noise, and merge the data. Chimeric sequences were removed using the consensus method.
Taxonomic classification was assigned using the SILVA database (ver. 132), which was specifically for the V4 region of the 16S rRNA gene. All taxonomic classifications were implemented within QIIME2 and assigned using the naïve Bayesian algorithm. For phylogenetic diversity analysis, sequences were aligned using the MAFFT program plugin [20] and used to construct a phylogenetic tree with FastTree 2 [21]. Alpha diversity (Chao richness, Shannon diversity, and Pielou evenness) was estimated using q2-diversity.

\section{Quantification of fecal and cecal IgA concentrations}

The feces collected after the 6-week pre-feeding period and the cecal content were resuspended in phosphate-buffered saline (PBS) and homogenized using a vortex mixer. The IgA concentration was measured in the fecal and cecal supernatants using a Mouse IgA enzyme-linked immunosorbent assay (ELISA) quantification kit (Bethyl Laboratories, Montgomery, TX, USA), according to the manufacturer's instructions. The absorbance of each well was read at $450 \mathrm{~nm}$ using a microplate reader (Bio-Rad Japan, Tokyo, Japan).

\section{Evaluation of ratio of IgA-coated microbiota in the cecal content}

The ratio of IgA-coated microbiota to total microbiota (IgA coating ratio) in the cecal content was measured as previously described [22]. Briefly, $100 \mu \mathrm{g}$ of cecal content was dissolved in $900 \mu \mathrm{L} \mathrm{PBS}$, and the cecal suspension was centrifuged at 100 $\times g$ for 20 min to remove debris. The supernatant was further centrifuged at $9,000 \times g$ for $10 \mathrm{~min}$. After centrifugation, the resultant bacterial pellet was washed twice with PBS. The bacterial cells were then fixed with $4 \%$ paraformaldehyde (Wako Pure Chemical) overnight at $4^{\circ} \mathrm{C}$. After washing twice with PBS, the bacterial cells were stained with FITC-labelled anti-mouse IgA (BD Pharmingen Japan, Tokyo, Japan) for $30 \mathrm{~min}$. After washing twice with PBS, the bacterial cells were stained with propidium iodide (PI; Sigma Aldrich Japan) solution $(4 \mu \mathrm{g} / \mathrm{mL})$. The bacterial suspension was loaded onto a Gallios flow cytometer (Beckman Coulter). Total microbiota and IgA-coated microbiota were detected as PI-positive populations and FITC/PI-double positive populations, respectively. FCS Express V3 (De Novo Software, Los Angeles, CA, USA) was used to calculate the IgA coating ratio.

\section{Statistical analysis}

Data were expressed as the mean \pm standard error of the mean (SEM). All statistical analyses were performed in GraphPad Prism version 7.00 for Windows (GraphPad Software, San Diego, CA, USA). Bartlett's test was used to assess homogeneity of variances across groups. For datasets with equal variances, group data were analyzed using one-way ANOVA with a post-hoc Tukey's test. For nonparametric data, Kruskal-Wallis analysis was used

Table 2. Criteria for histological scoring

\begin{tabular}{cl}
\hline Score & \\
\hline 0 & No evidence of inflammation. \\
1 & Low level of inflammation with scattered infiltrating mononuclear cells and neutrophils. \\
2 & Moderate inflammation with multiple foci of neutrophils. \\
3 & High degree of inflammation with increased vascular density and marked wall thickening. \\
4 & Maximal severity of inflammation with transmural leukocyte infiltration and loss of goblet cells accompanied by ulceration.
\end{tabular}


with Dunn's multiple comparison test. $\mathrm{P}<0.05$ was considered statistically significant.

\section{RESULTS}

\section{Body weight change, disease activity index, and colon length}

Chemically induced colitis was observed 7 days post-DSS administration and was characterized by significant loss of body weight, decreased stool consistency, and increased rectal bleeding (Fig. 1A and 1B). Body weight was significantly lower in the DSS and CNN-DSS groups than in the control group at days 10 and 14; however, there were no significant differences in the body weights between the DSS and CNN-DSS groups (Fig. 1A). On days 7 and 14 , the DAI score was significantly lower in the CNN-DSS group compared with that in the DSS group (Fig. 1B). The colon length was significantly reduced after DSS treatment, but there were no significant differences between the DSS and CNN-DSS groups (Fig. 1C).

\section{Gene expression of inflammatory cytokines in the colon}

The mRNA expression levels of the inflammatory cytokine genes $i l 1 b$, il6, and $m c p l$ in the colon are shown in Fig. 2A. $1 l 1 b$ and il6 expression levels were significantly higher in the DSS group compared with those in the control group. $I l 1 b$ expression was significantly reduced in the CNN-DSS group compared with that in the DSS group. No significant differences were observed in $I l 6$ expression between the DSS and CNN-DSS groups. The expression of $m c p 1$ tended to be lower in the CNN-DSS group compared with that in the DSS group $(\mathrm{p}=0.0823)$.

\section{MPO activity in the colon}

MPO activity in the colon is shown in Fig. 2B. MPO activity significantly increased in the DSS group compared with that in the control group. MPO activity tended to be lower in the CNN-DSS group compared with that in the DSS group $(\mathrm{p}=0.1703)$.

\section{Histological examination of colonic tissue}

The distal colon of the DSS group presented with marked crypt destruction, infiltration of multiple inflammatory cells, and loss of goblet cells. Although the histological score was significantly higher in the DSS and CNN-DSS groups compared with that in the control group, the CNN-DSS group showed a significantly lower histological score compared with the DSS group due to the reduced infiltration of immune cells and loss of goblet cells (Fig. 3A and 3B). Alcian Blue staining revealed that the goblet cell number per crypt was significantly decreased in the DSS and CNN-DSS groups compared with that in the control group. Interestingly, however, the decrease induced by DSS was ameliorated by CNN treatment (Fig. 3C and 3D).

\section{Phylogenetic diversity of cecal microbiota}

The composition of microbial communities was analyzed by calculating three major ecological parameters: Chao richness (an estimate of a total community), Pielou evenness (a measure of how evenly the individuals within a community are distributed over different operational taxonomic units), and Shannon diversity (the combined parameter for richness and evenness). Although these ecological parameters decreased in the DSS group compared with those in the control group, there were no significant differences among the experimental groups (Fig. 4).
A

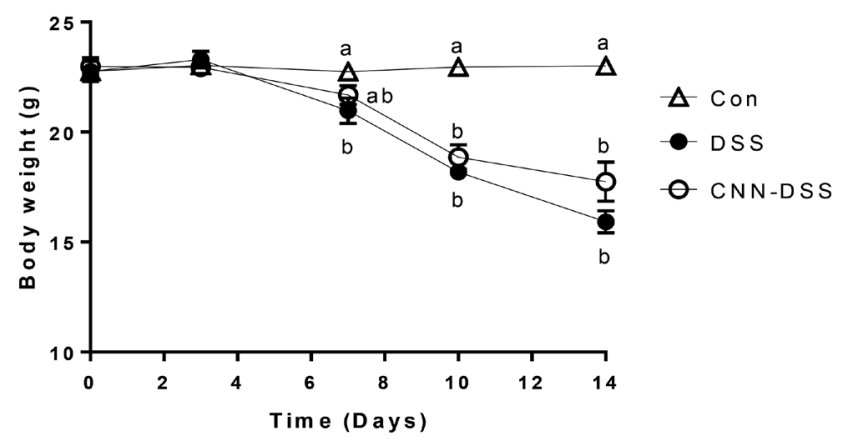

B

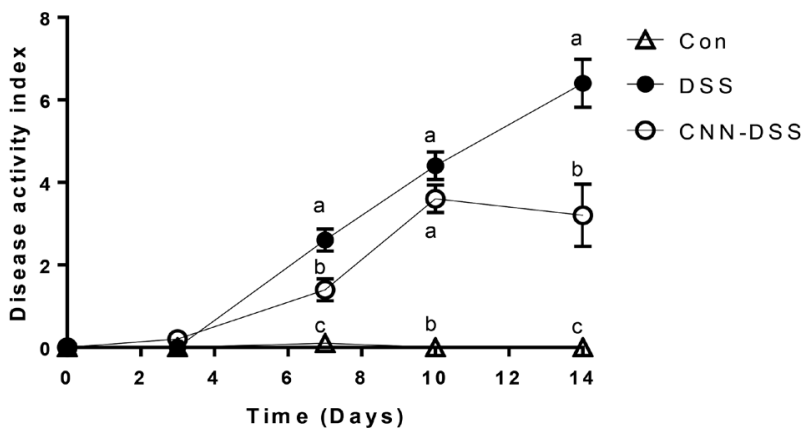

C

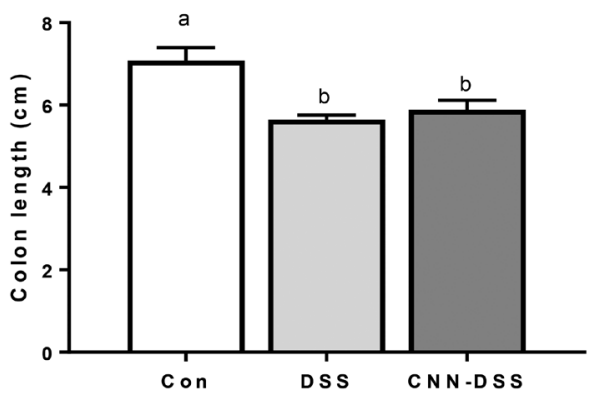

Fig. 1. Effects of cyclic nigerosylnigerose $(\mathrm{CNN})$ on body weight, disease activity index, and colon length in mice with DSS-induced colitis. (A) Body weight. (B) Disease activity index. (C) Colon length. Con: control group; DSS: DSS group; CNN-DSS: CNN-DSS group. Data are expressed as the mean \pm SEM. Values without a common letter are statistically significantly different $(p<0.05)$.

\section{Relative abundance of cecal microbiota}

The microbial composition at the phylum level is shown in Fig. $5 \mathrm{~A}$. The relative abundance of Bacteroidetes tended to be higher in the DSS group $(44.46 \pm 5.62 \%)$ compared with that in the control $(34.11 \pm 3.48 \%)$ and CNN-DSS groups $(35.21 \pm 5.82 \%)$. The relative abundance of Firmicutes tended to be lower in the DSS group $(49.07 \pm 4.94 \%)$ compared with that in the control $(58.48 \pm 2.74 \%)$ and CNN-DSS groups $(57.75 \pm 4.48 \%)$ (Fig. $5 \mathrm{~A})$, although there were no significant differences in any of the phyla among the experimental groups. The microbial composition at the family level is shown in Fig. 5B. There were no significant differences in the relative abundances of any families among the experimental groups. The relative abundances of microbial taxa that were significantly different among experimental groups are shown in Fig. 5C. The relative abundance of the Lachnospiraceae NK4A136 group was significantly higher in the CNN-DSS group 

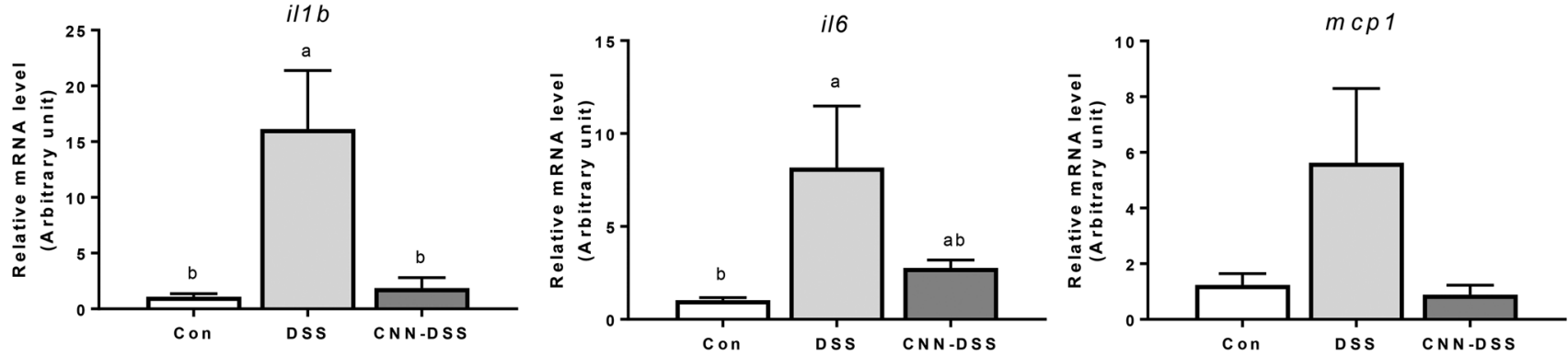

B

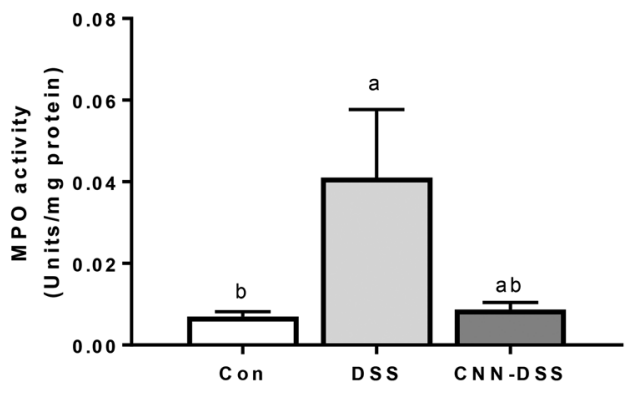

compared with that in the DSS group, but not that in the control group. The relative abundance of Muribaculaceae unclassified genus A was significantly higher in the control group compared with that in the DSS and CNN-DSS groups. The relative abundance of Muribaculaceae unclassified genus B was significantly higher in the DSS and CNN-DSS groups compared with that in the control group.

\section{Fecal and cecal IgA concentrations}

The IgA concentrations in the feces collected after 6 weeks of $\mathrm{CNN}$ pre-feeding and cecal content on day 14 post-DSS treatment are shown in Fig. 6A and 6B, respectively. CNN pre-feeding for 6 weeks significantly increased the fecal IgA concentration compared with the control group (Fig. 6A). After DSS treatment, there were no significant differences in the cecal IgA concentration among the experimental groups (Fig. 6B).

\section{IgA coating ratio in the cecal content}

Representative results of flow cytometry for the detection of IgA-coated bacteria in the cecal content are shown in Fig. $6 \mathrm{C}$. A quadrant gate was drawn using the single-color controls to determine FITC- and PI-positive populations. IgA-coated bacteria are located within the upper right quadrant in the dot plot as a FITC- and PI-positive population. The IgA coating ratio in the cecal content tended to be higher in the CNN-DSS group compared with those in the control group $(\mathrm{p}=0.0566)$ and DSS group ( $p=0.0705$ ) (Fig. 6D), although there was no difference between the control and DSS groups.

\section{DISCUSSION}

DSS-induced colitis is a gut inflammation model that resembles human ulcerative colitis [23]. Although the etiology of IBDs is not fully understood, some reports have suggested that an aberrant immune response against gut microbiota might be a major cause of gut inflammation [24]. Therefore, factors that suppress excessive inflammatory responses against commensal microbiota in the gut have been thought to prevent the development of IBDs. CNN is a cyclic oligosaccharide that can promote IgA secretion in the gut. Previous studies have suggested that by coating gut microbiota, IgA may be important for establishing a symbiotic relationship with gut microbiota without inducing gut inflammation [13, 14]. In the current study, we examined the effect of oral administration of $\mathrm{CNN}$ on severity of colitis, inflammatory state in the colon, colonic histology, goblet cell number, cecal microbiota composition, IgA concentration, and IgA coating ratio in DSS-induced colitis mice.

The DAI score was significantly decreased in the CNN-DSS group compared with the DSS group. Consistent with the DAI score, the DSS-induced increase in $i l 1 b$ expression in the colon was significantly reduced by CNN treatment. IL-1 $\beta$ is mainly produced by activated macrophages and plays a vital role in the incidence of IBD [25]. Our results suggest that CNN treatment might alleviate DSS-induced gut inflammation by inhibiting the activation of macrophages in the colon. Despite amelioration of the DAI score and colonic $i l 1 b$ gene expression in the CNNDSS group, the reduction of colon length induced by DSS was not recovered by CNN treatment. The cause of this discrepancy remains unclear.

MPO is a member of the heme peroxidase superfamily and is mainly found in neutrophils. Therefore, colonic MPO activity is a measure of neutrophil infiltration into inflamed tissue and is considered to be a marker of tissue damage [26]. The increased MPO activity induced by DSS was ameliorated by CNN treatment, indicating that infiltration of neutrophils into the colonic mucosa might be suppressed by CNN treatment. This speculation is supported by the significantly lower histological score in the CNN-DSS group compared with that in the DSS group due to decreased infiltration of immune cells.

Mucus is continuously secreted by colonic goblet cells and is mainly composed of heavily $\mathrm{O}$-glycosylated proteins called mucins $[27,28]$. Mucus layers play an important role in separating gut microbiota and colonic epithelial cells [29]. In this study, 
A

Con

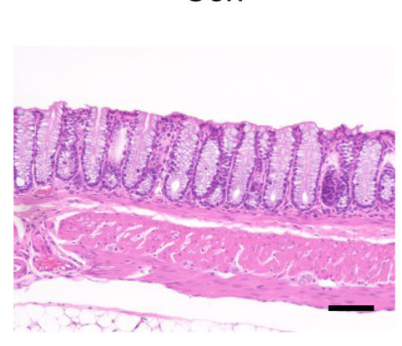

C

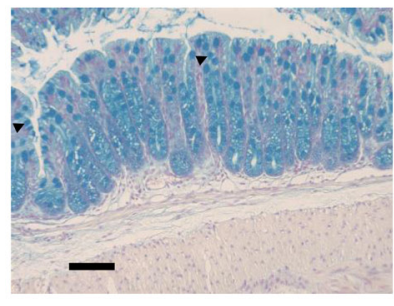

DSS

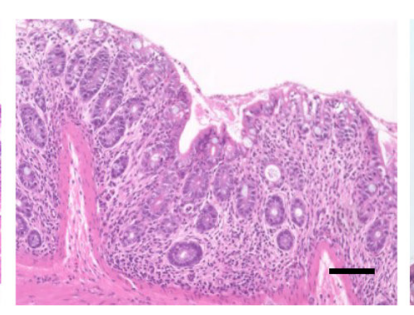

DSS

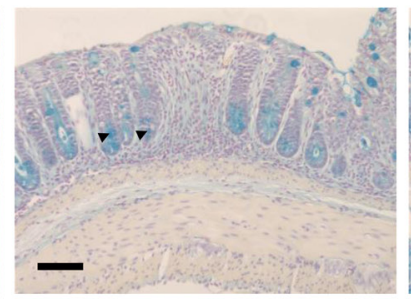

CNN-DSS

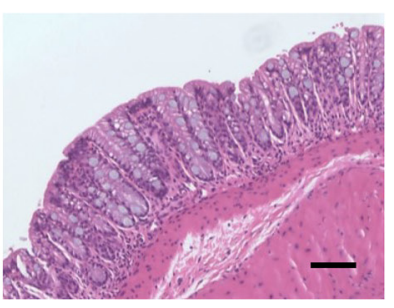

CNN-DSS

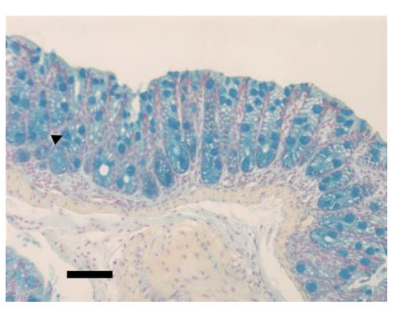

B

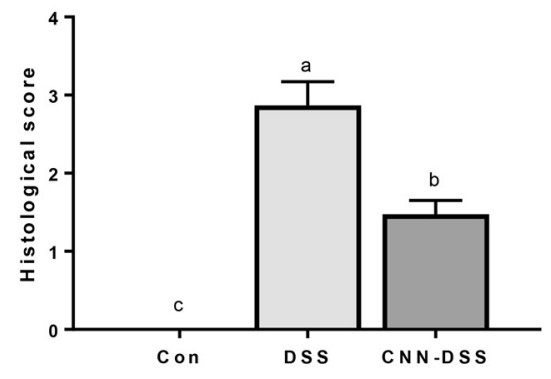

D

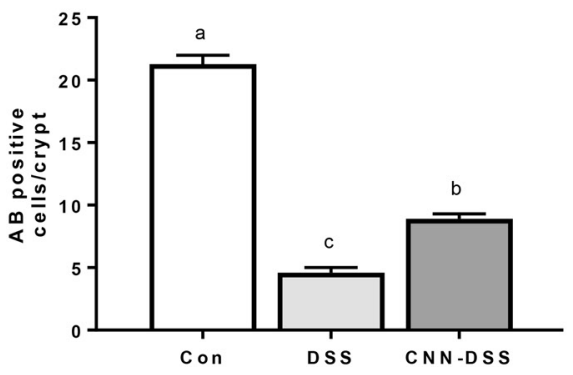

Fig. 3. Effects of cyclic nigerosylnigerose $(\mathrm{CNN})$ on the histology and the number of goblet cells in the colonic tissue of mice with DSS-induced colitis. (A) Representative images of H\&E-stained colonic tissues. (B) Histological score. (C) Representative images of colonic tissues stained with Alcian Blue (AB). (D) Number of AB-positive cells. AB-positive cells are indicated by arrows. Con: control group; DSS: DSS group; CNN-DSS: CNN-DSS group. Data are expressed as the mean \pm SEM. Each section is shown at $200 \times$ magnification. The black scale bar indicates $100 \mu \mathrm{m}$ for all panels. Values without a common letter are statistically significantly different $(\mathrm{p}<0.05)$.

A

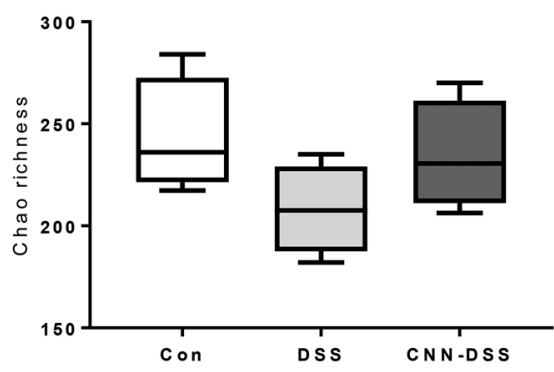

B

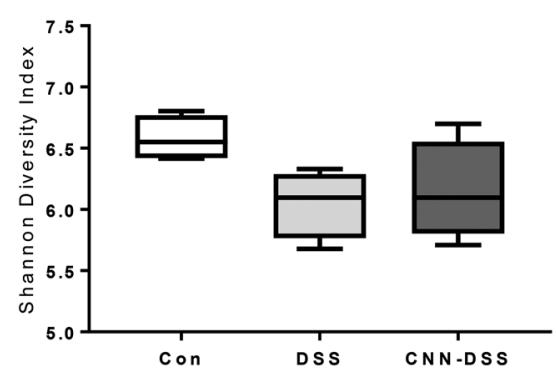

C

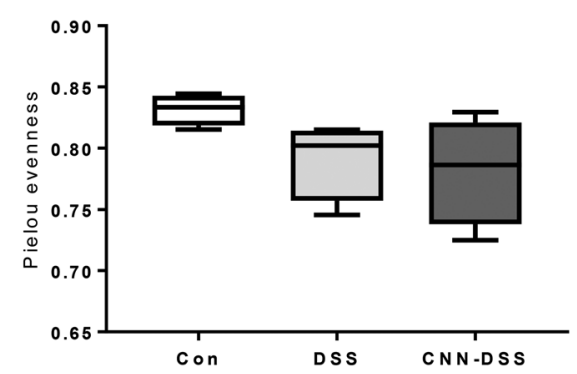

Fig. 4. Effects of cyclic nigerosylnigerose $(\mathrm{CNN})$ on the phylogenetic diversity of cecal microbiota in mice with DSS-induced colitis. (A) Chao richness, (B) Shannon diversity, and (C) Pielou evenness. Data are expressed as the mean \pm SEM. Con: control group; DSS: DSS group; CNN-DSS: CNN-DSS group.

DSS-induced loss of goblet cells was partly restored by CNN treatment. Similar results were observed for other food ingredients and probiotic bacteria. Wang et al. showed that dietary grape seed extract improved gut inflammation with an increase in the colonic goblet cell number in IL10-deficient mice, which spontaneously developed colitis [30]. Moreover, oral administration of Bifidobacterium longum CCM7952 suppressed the loss of goblet cells and ameliorated the symptoms of DSS-induced colitis in mice [31]. Our observations suggested that since $\mathrm{CNN}$ is able to preserve goblet cells in the colon, it may play a crucial role in the prevention of gut inflammation.

Previous studies have shown that the number of goblet cells in the colon is affected by gut microbiota. Wrzosek et al. showed that goblet cell differentiation was promoted in Bacteroides thetaiotaomicron mono-associated mice but was suppressed by Faecalibacterium prausnitzii [32]. Furthermore, oral administration of grape seed extract has been shown to increase the number of goblet cells in IL-10-deficient colitis mice while increasing the relative abundance of the genus Bacteroides and decreasing that of $F$. prausnitzii [30]. In this study, we compared the relative abundances of $B$. thetaiotaomicron and $F$. prausnitzii in the cecal content among the groups by quantitative PCR using specific primer pairs. There were no significant differences in the relative abundances of $B$. thetaiotaomicron and $F$. prausnitzii among the groups (data not shown). Our results suggest that CNN might restore the loss of goblet cells via mechanisms other than the modification of gut microbial composition. These mechanisms should be the focus of future studies. 
A

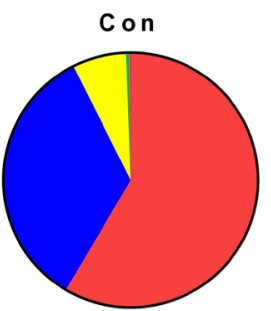

B

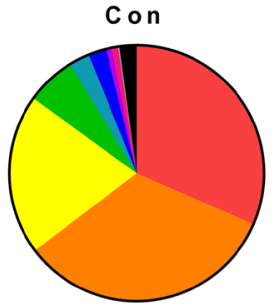

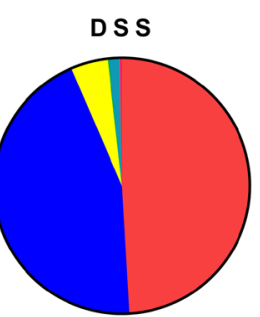
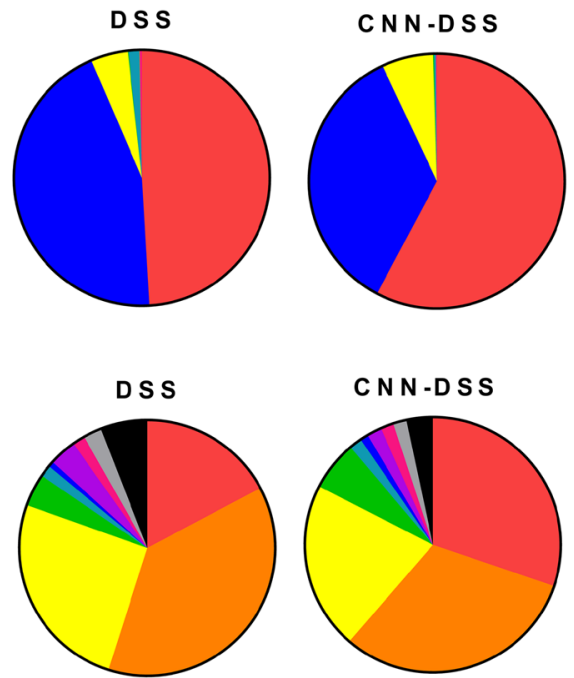

C

Lachnospiraceae NK4A136 group
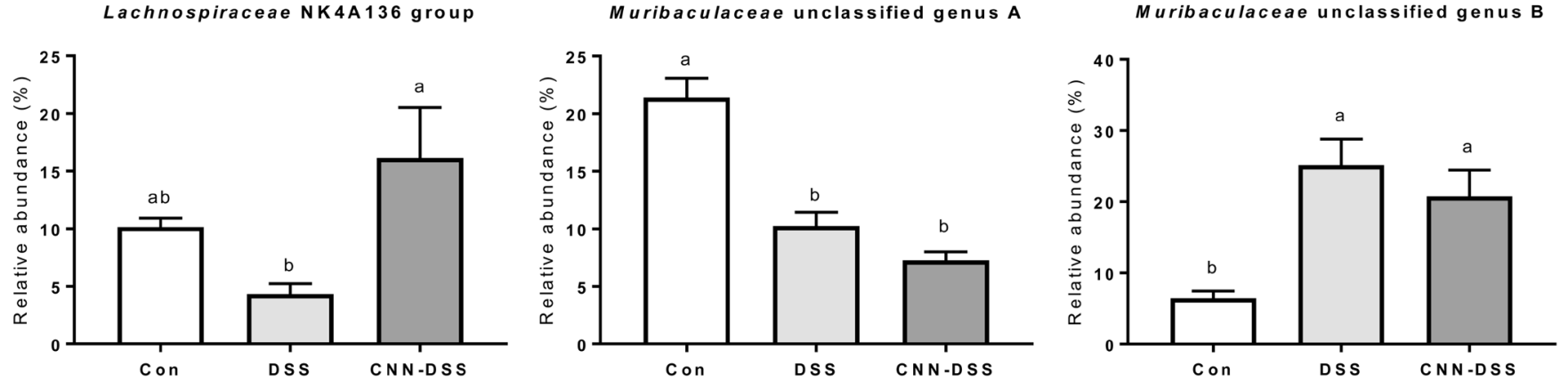

Fig. 5. Effects of cyclic nigerosylnigerose (CNN) on the composition of cecal microbiota in mice with DSS-induced colitis. Pie chart representation of relative abundances of cecal microbiota (A) at the phylum and (B) family levels. (C) Relative abundances of cecal microbiota at the genus level that were significantly different among experimental groups. Con: control group; DSS: DSS group; CNN-DSS: CNN-DSS group. Data are expressed as the mean \pm SEM. Values without a common letter are statistically significantly different $(\mathrm{p}<0.05)$.

Changes in the composition of gut microbiota have been linked to the pathogenesis of IBD [33]. Three ecological parameters, Chao richness, Pielou evenness, and Shannon diversity, were reduced in mice with DSS-induced colitis and in IBD patients [33, 34]. In this study, although these parameters seemed to be reduced by DSS treatment, which was consistent with previous studies, DSS-induced changes in these parameters were not restored by CNN treatment. These data suggest that CNN treatment might not influence the richness, evenness, and diversity of microbial communities.

Praengam et al. showed that oral administration of brown rice alleviated gut inflammation in mice with DSS-induced colitis and reversed DSS-induced changes in gut microbial composition, such as decreased Firmicutes and increased Bacteroidetes [4]. Similar to this study, CNN treatment tended to change the gut microbial composition in DSS-induced colitis mice at the phylum level. Our study also revealed that the CNN-DSS group showed a significantly higher relative abundance of Lachnospiraceae NK4A136 compared with the DSS group despite there being no difference in cecal total bacteria among all groups (data not shown). $\mathrm{Hu}$ et al. suggested that the Lachnospiraceae NK4A136 group might play a pivotal role in suppressing chronic inflammation in high-fat diet-induced obese mice by producing butyrate [35].
Short-chain fatty acids, mainly consisting of acetate, propionate, and butyrate, are metabolites of gut microbiota, which can inhibit inflammatory responses [36, 37]. Our observation suggests that a shift in Lachnospiraceae NK4A136 induced by CNN may partly contribute to its anti-inflammatory effect.

The IgA coating ratio in the cecal content tended to be higher in the CNN-DSS group compared with those in the control and DSS groups. This result suggests that CNN treatment might promote IgA coating of gut microbiota. There are three possible mechanisms for the increase of the IgA coating ratio induced by $\mathrm{CNN}$ treatment. The first involves the modification of the microbial composition by $\mathrm{CNN}$ treatment, which is to say that the abundance of certain gut microbiota, preferentially coated with IgA, might increase in the CNN-DSS group. The second possible mechanism is related to the restoration of the DSS-induced loss of goblet cells by CNN treatment. IgA is anchored to the outer layer of colonic mucus, possibly via the glycan-dependent association of secretory components with mucus proteins [38, 39]. In the mucus layer, IgA is associated with gut microbiota [38, 40]. These findings suggest that the IgA-mucus complex might be crucial for efficient IgA coating of gut microbiota. The restoration of goblet cells induced by $\mathrm{CNN}$ treatment might contribute to the formation of a complex of $\operatorname{IgA}$, mucus, and microbiota, which 
A

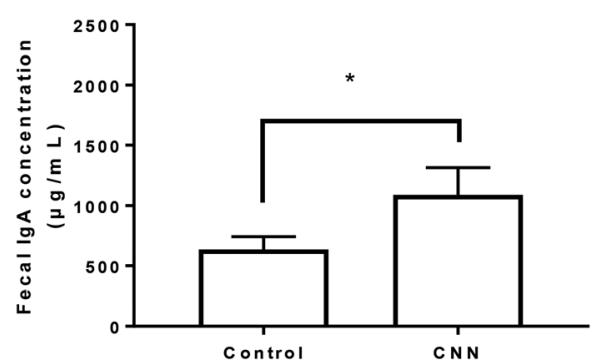

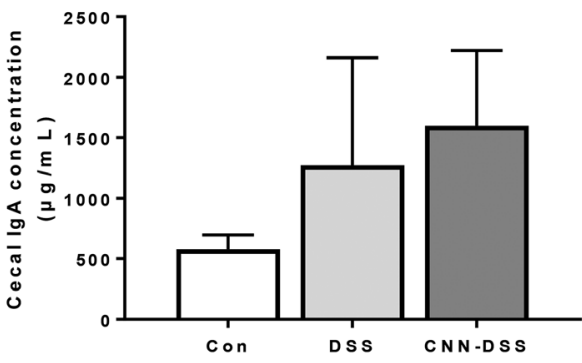

C
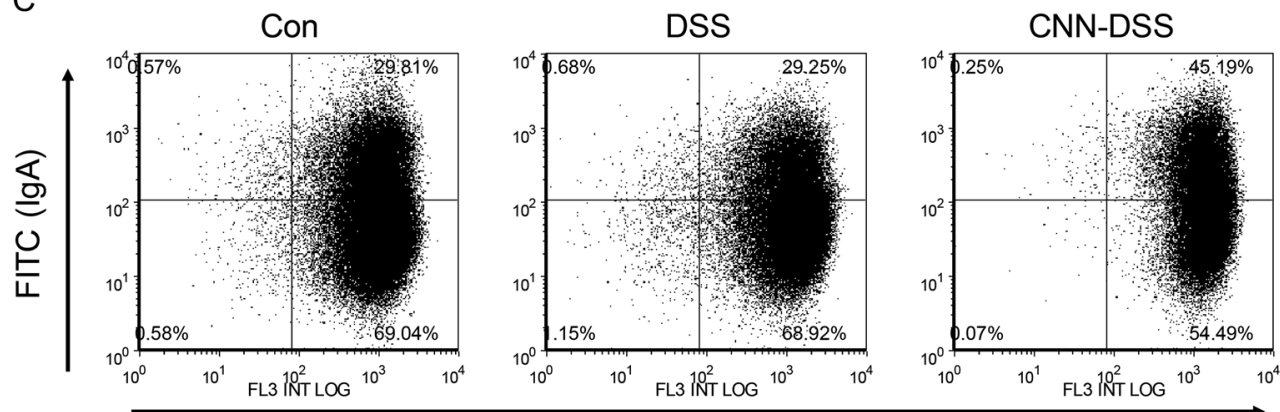

Propidium iodide (Gut microbiota)

D

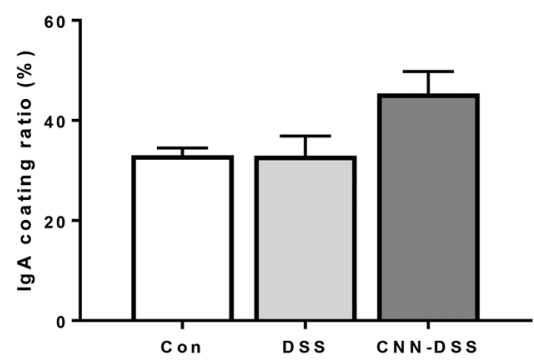

Fig. 6. Effects of cyclic nigerosylnigerose $(\mathrm{CNN})$ on the $\operatorname{IgA}$ concentration and the IgA reactivity against gut microbiota in the cecal content of mice with DSS-induced colitis. IgA concentration (A) in the feces collected after 6 weeks of CNN pre-feeding and (B) cecal content on day 14 post-DSS treatment. (C) Representative results of flow cytometry for the detection of IgA-coated bacteria. A quadrant gate was drawn to determine FITC- and PI-positive populations. IgA-coated bacteria are located within the upper right quadrant in the dot plot as a FITC- and PI-positive population. (D) IgA coating ratio in the cecal content on day 14 post-DSS treatment. Con: control group; DSS: DSS group; CNN-DSS: CNN-DSS group. Data are expressed as the mean \pm SEM. *Statistically significantly different $(p<0.05)$.

might have resulted in the increased IgA coating ratio in the CNN-DSS group. The third possible mechanism is an increase in IgA reactivity against gut microbiota.

Based on our study, it remains unclear whether the effect of $\mathrm{CNN}$ on IgA coating of gut microbiota is related to symptoms of DSS-induced colitis. Previous studies have revealed that specific IgA antibodies against gut microbiota are essential to suppress the growth of gut microbiota [14, 41]. Boullier et al. reported that monoclonal IgA specific for the pathogenic bacterium Shigella flexneri preferentially binds to mucus layers and restricts the invasion of $S$. flexneri into the submucosal area [42]. Furthermore, IgA has also been shown to preferentially coat inflammation-inducible and invasive gut microbiota to prevent gut inflammation [43]. These reports suggest that increased IgA coating of gut microbiota induced by $\mathrm{CNN}$ may be partly related to the effect of $\mathrm{CNN}$ in altering gut microbial composition and suppressing gut inflammation.

In conclusion, CNN treatment reduced gut inflammation in mice with DSS-induced colitis, possibly through synergistic effects of restoration of goblet cells, increased abundance of butyrate-producing bacteria, and promotion of $\operatorname{IgA}$ coating of gut microbiota. Furthermore, this study raises the possibility that IgA reactivity against gut microbiota may be modulated by dietary ingredients. The IgA coating of gut microbiota may be a useful marker for identifying beneficial food ingredients that prevent gut inflammation.

\section{CONFLICTS OF INTEREST}

None declared.

\section{ACKNOWLEDGMENT}

We would like to thank the Assisted Reproductive Technology Center and the Department of Genomics and Proteomics at Okayama University for their technical support. 


\section{REFERENCES}

1. Maloy KJ, Powrie F. 2011. Intestinal homeostasis and its breakdown in inflammatory bowel disease. Nature 474: 298-306. [Medline] [CrossRef]

2. Manichanh C, Borruel N, Casellas F, Guarner F. 2012. The gut microbiota in IBD. Nat Rev Gastroenterol Hepatol 9: 599-608. [Medline] [CrossRef]

3. Podolsky DK. 1991. Inflammatory bowel disease (2). N Engl J Med 325: 1008-1016. [Medline] [CrossRef]

4. Praengam K, Sahasakul Y, Kupradinun P, Sakarin S, Sanitchua W, Rungsipipat A, Rattanapinyopituk K, Angkasekwinai P, Changsri K, Mhuantong W, Tangphatsornruang S, Tuntipopipat S. 2017. Brown rice and retrograded brown rice alleviate inflammatory response in dextran sulfate sodium (DSS)-induced colitis mice. Food Funct 8: 46304643. [Medline] [CrossRef]

5. Watanabe H, Nakano M, Oku K, Aga H, Nishimoto T, Kubota M, Fukuda S, Kurimoto M, Tsujisaka Y. 2004. Cyclic tetrasaccharide in sake lees. J Appl Glycosci 51: 345-347. [CrossRef]

6. Aga H, Higashiyama T, Watanabe H, Sonoda T, Nishimoto T, Kubota M, Fukuda S, Kurimoto M, Tsujisaka Y. 2002. Production of cyclic tetrasaccharide from starch using a novel enzyme system from Bacillus globisporus C11. J Biosci Bioeng 94: 336-342. [Medline] [CrossRef]

7. Aga H, Nishimoto T, Kuniyoshi M, Maruta K, Yamashita H, Higashiyama T, Nakada T, Kubota M, Fukuda S, Kurimoto M, Tsujisaka Y. 2003. 6- $\alpha$-glucosyltransferase and 3- $\alpha$-isomaltosyltransferase from Bacillus globisporus N75. J Biosci Bioeng 95: 215-224. [Medline] [CrossRef]

8. Hino K, Kurose M, Sakurai T, Inoue S, Oku K, Chaen H, Kohno K, Fukuda S. 2006. Effect of dietary cyclic nigerosylnigerose on intestinal immune functions in mice. Biosci Biotechnol Biochem 70: 2481-2487. [Medline] [CrossRef]

9. Macpherson AJ, Geuking MB, McCoy KD. 2011. Immunoglobulin A: a bridge between innate and adaptive immunity. Curr Opin Gastroenterol 27: 529-533. [Medline] [CrossRef]

10. Mantis NJ, Rol N, Corthésy B. 2011. Secretory IgA's complex roles in immunity and mucosal homeostasis in the gut. Mucosal Immunol 4: 603-611. [Medline] [CrossRef]

11. Pabst O. 2012. New concepts in the generation and functions of IgA. Nat Rev Immunol 12: 821-832. [Medline] [CrossRef]

12. Cullender TC, Chassaing B, Janzon A, Kumar K, Muller CE, Werner JJ, Angenent LT, Bell ME, Hay AG, Peterson DA, Walter J, Vijay-Kumar M, Gewirtz AT, Ley RE. 2013. Innate and adaptive immunity interact to quench microbiome flagellar motility in the gut. Cell Host Microbe 14: 571-581. [Medline] [CrossRef]

13. Kubinak JL, Petersen C, Stephens WZ, Soto R, Bake E, O'Connell RM, Round JL. 2015. MyD88 signaling in T cells directs IgA-mediated control of the microbiota to promote health. Cell Host Microbe 17: 153-163. [Medline] [CrossRef]

14. Peterson DA, McNulty NP, Guruge JL, Gordon JI. 2007. IgA response to symbiotic bacteria as a mediator of gut homeostasis. Cell Host Microbe 2: 328-339. [Medline] [CrossRef]

15. Murthy SN, Cooper HS, Shim H, Shah RS, Ibrahim SA, Sedergran DJ. 1993. Treatment of dextran sulfate sodium-induced murine colitis by intracolonic cyclosporin. Dig Dis Sci 38: 1722-1734. [Medline] [CrossRef]

16. Kim JJ, Shajib MS, Manocha MM, Khan WI. 2012. Investigating intestinal inflammation in DSS-induced model of IBD. J Vis Exp: 3678. [Medline]

17. Albert E, Walker J, Thiesen A, Churchill T, Madsen K. 2010. cis-Urocanic acid attenuates acute dextran sodium sulphate-induced intestinal inflammation. PLoS One 5: e13676. [Medline] [CrossRef]

18. Bolyen E, Rideout JR, Dillon MR, Bokulich NA, Abnet CC, Al-Ghalith GA, Alexander H, Alm EJ, Arumugam M, Asnicar F, Bai Y, Bisanz JE, Bittinger K, Brejnrod A, Brislawn CJ, Brown CT, Callahan BJ, Caraballo-Rodríguez AM, Chase J, Cope EK, Da Silva R, Diener C, Dorrestein PC, Douglas GM, Durall DM, Duvallet C, Edwardson CF, Ernst M, Estaki M, Fouquier J, Gauglitz JM, Gibbons SM, Gibson DL, Gonzalez A, Gorlick K, Guo J, Hillmann B, Holmes S, Holste H, Huttenhower C, Huttley GA, Janssen S, Jarmusch AK, Jiang L, Kaehler BD, Kang KB, Keefe CR, Keim P, Kelley ST, Knights D, Koester I, Kosciolek T, Kreps J, Langille MGI, Lee J, Ley R, Liu YX, Loftfield E, Lozupone C, Maher M, Marotz C, Martin BD, McDonald D, McIver LJ, Melnik AV, Metcalf JL, Morgan SC, Morton JT, Naimey AT, Navas-Molina JA, Nothias LF, Orchanian SB, Pearson T, Peoples SL, Petras D, Preuss ML, Pruesse E, Rasmussen LB, Rivers A, Robeson MS 2nd, Rosenthal P, Segata N, Shaffer M, Shiffer A, Sinha R, Song SJ, Spear JR, Swafford AD, Thompson LR, Torres PJ, Trinh P, Tripathi A, Turnbaugh PJ, Ul-Hasan S, van der Hooft JJJ, Vargas F, Vázquez-Baeza Y, Vogtmann E, von Hippel M, Walters W, Wan Y, Wang M, Warren J, Weber KC, Williamson CHD, Willis AD, Xu ZZ, Zaneveld JR, Zhang Y, Zhu Q, Knight R, Caporaso JG. 2019. Reproducible, interactive, scalable and extensible microbiome data science using QIIME 2. Nat Biotechnol 37: 852-857. [Medline] [CrossRef]

19. Callahan BJ, McMurdie PJ, Rosen MJ, Han AW, Johnson AJA, Holmes SP. 2016. DADA2: high-resolution sample inference from Illumina amplicon data. Nat Methods 13: 581-583. [Medline] [CrossRef]

20. Katoh K, Misawa K, Kuma K, Miyata T. 2002. MAFFT: a novel method for rapid multiple sequence alignment based on fast Fourier transform. Nucleic Acids Res 30:
3059-3066. [Medline] [CrossRef]

21. Price MN, Dehal PS, Arkin AP. 2010. FastTree 2 - approximately maximum-likelihood trees for large alignments. PLoS One 5: e9490. [Medline] [CrossRef]

22. Muhomah TA, Nishino N, Katsumata E, Haoming W, Tsuruta T. 2019. High-fat diet reduces the level of secretory immunoglobulin A coating of commensal gut microbiota. Biosci Microbiota Food Health 38: 55-64. [Medline] [CrossRef]

23. Jiminez JA, Uwiera TC, Douglas Inglis G, Uwiera RR. 2015. Animal models to study acute and chronic intestinal inflammation in mammals. Gut Pathog 7: 29. [Medline] [CrossRef]

24. Sartor RB. 2006. Mechanisms of disease: pathogenesis of Crohn's disease and ulcerative colitis. Nat Clin Pract Gastroenterol Hepatol 3: 390-407. [Medline] [CrossRef]

25. Kwon KH, Murakami A, Hayashi R, Ohigashi H. 2005. Interleukin-1 $\beta$ targets interleukin-6 in progressing dextran sulfate sodium-induced experimental colitis. Biochem Biophys Res Commun 337: 647-654. [Medline] [CrossRef]

26. Palmen MJ, Dijkstra CD, van der Ende MB, Peña AS, van Rees EP. 1995. Anti-CD11b/ CD18 antibodies reduce inflammation in acute colitis in rats. Clin Exp Immunol 101: 351-356. [Medline] [CrossRef]

27. Birchenough GM, Johansson ME, Gustafsson JK, Bergström JH, Hansson GC. 2015 New developments in goblet cell mucus secretion and function. Mucosal Immunol 8: 712-719. [Medline] [CrossRef]

28. Kim YS, Ho SB. 2010. Intestinal goblet cells and mucins in health and disease: recent insights and progress. Curr Gastroenterol Rep 12: 319-330. [Medline] [CrossRef]

29. Johansson ME, Phillipson M, Petersson J, Velcich A, Holm L, Hansson GC. 2008. The inner of the two Muc2 mucin-dependent mucus layers in colon is devoid of bacteria. Proc Natl Acad Sci USA 105: 15064-15069. [Medline] [CrossRef]

30. Wang H, Xue Y, Zhang H, Huang Y, Yang G, Du M, Zhu MJ. 2013. Dietary grape seed extract ameliorates symptoms of inflammatory bowel disease in IL10-deficient mice. Mol Nutr Food Res 57: 2253-2257. [Medline] [CrossRef]

31. Srutkova D, Schwarzer M, Hudcovic T, Zakostelska Z, Drab V, Spanova A, Rittich B, Kozakova H, Schabussova I. 2015. Bifidobacterium longum CCM 7952 promotes epithelial barrier function and prevents acute DSS-induced colitis in strictly strainspecific manner. PLoS One 10: e134050. [Medline] [CrossRef]

32. Wrzosek L, Miquel S, Noordine ML, Bouet S, Joncquel Chevalier-Curt M, Robert V, Philippe C, Bridonneau C, Cherbuy C, Robbe-Masselot C, Langella P, Thomas M. 2013. Bacteroides thetaiotaomicron and Faecalibacterium prausnitzii influence the production of mucus glycans and the development of goblet cells in the colonic epithelium of a gnotobiotic model rodent. BMC Biol 11: 61. [Medline] [CrossRef]

33. Walker AW, Sanderson JD, Churcher C, Parkes GC, Hudspith BN, Rayment N, Brostoff J, Parkhill J, Dougan G, Petrovska L. 2011. High-throughput clone library analysis of the mucosa-associated microbiota reveals dysbiosis and differences between inflamed and non-inflamed regions of the intestine in inflammatory bowel disease. BMC Microbiol 11: 7. [Medline] [CrossRef]

34. Rodríguez-Nogales A, Algieri F, Garrido-Mesa J, Vezza T, Utrilla MP, Chueca N Fernández-Caballero JA, García F, Rodríguez-Cabezas ME, Gálvez J. 2018. The administration of Escherichia coli Nissle 1917 ameliorates development of DSSinduced colitis in mice. Front Pharmacol 9: 468. [Medline] [CrossRef]

35. Hu S, Wang J, Xu Y, Yang H, Wang J, Xue C, Yan X, Su L. 2019. Anti-inflammation effects of fucosylated chondroitin sulphate from Acaudina molpadioides by altering gut microbiota in obese mice. Food Funct 10: 1736-1746. [Medline] [CrossRef]

36. Sircana A, Framarin L, Leone N, Berrutti M, Castellino F, Parente R, De Michieli F, Paschetta E, Musso G. 2018. Altered gut microbiota in type 2 diabetes: just a coincidence? Curr Diab Rep 18: 98. [Medline] [CrossRef]

37. Tan J, McKenzie C, Potamitis M, Thorburn AN, Mackay CR, Macia L. 2014. The role of short-chain fatty acids in health and disease. Advances in immunology, Elsevier.

38. Rogier EW, Frantz AL, Bruno ME, Kaetzel CS. 2014. Secretory IgA is concentrated in the outer layer of colonic mucus along with gut bacteria. Pathogens 3: 390-403. [Medline] [CrossRef]

39. Phalipon A, Cardona A, Kraehenbuhl JP, Edelman L, Sansonetti PJ, Corthésy B. 2002. Secretory component: a new role in secretory IgA-mediated immune exclusion in vivo. Immunity 17: 107-115. [Medline] [CrossRef]

40. van der Waaij LA, Limburg PC, Mesander G, van der Waaij D. 1996. In vivo IgA coating of anaerobic bacteria in human faeces. Gut 38: 348-354. [Medline] [CrossRef]

41. Wei M, Shinkura R, Doi Y, Maruya M, Fagarasan S, Honjo T. 2011. Mice carrying a knock-in mutation of Aicda resulting in a defect in somatic hypermutation have impaired gut homeostasis and compromised mucosal defense. Nat Immunol 12: 264-270. [Medline] [CrossRef]

42. Boullier S, Tanguy M, Kadaoui KA, Caubet C, Sansonetti P, Corthésy B, Phalipon A. 2009. Secretory IgA-mediated neutralization of Shigella flexneri prevents intestinal tissue destruction by down-regulating inflammatory circuits. J Immunol 183: 58795885. [Medline] [CrossRef]

43. Palm NW, de Zoete MR, Cullen TW, Barry NA, Stefanowski J, Hao L, Degnan PH, Hu J, Peter I, Zhang W, Ruggiero E, Cho JH, Goodman AL, Flavell RA. 2014. Immunoglobulin A coating identifies colitogenic bacteria in inflammatory bowel disease. Cell 158: 1000-1010. [Medline] [CrossRef] 